\title{
Calculated properties of nitrogen-vacancy complexes in beryllium- and magnesium-doped GaN
}

\author{
C. D. Latham* and R. Jones \\ School of Physics, University of Exeter Exeter, EX4 4QL, United Kingdom \\ S. Öberg \\ Department of Mathematics, Lulea University of Technology, SE-97187 Luleå, Sweden \\ R. M. Nieminen \\ Laboratory of Physics, Helsinki University of Technology, P.O. Box 1100, FIN-02015 HUT, Finland \\ P. R. Briddon \\ Physics Centre, School of Natural Science, University of Newcastle upon Tyne, Newcastle NE1 7RU, United Kingdom
}

(Received 1 April 2003; revised manuscript received 1 July 2003; published 20 November 2003)

\begin{abstract}
The properties of defect complexes consisting of a nitrogen vacancy with a substitutional beryllium or magnesium atom on neighboring lattice sites in hexagonal $\mathrm{GaN}$ are calculated using the AIMPRO local-densityfunctional theory method. Both types of defects $V_{\mathrm{N}}-\mathrm{Be}_{\mathrm{Ga}}$ and $V_{\mathrm{N}}-\mathrm{Mg}_{\mathrm{Ga}}$ are bound with respect to their isolated constituents. They do not appear to have any electronic levels in the bandgap, and are expected to be neutral defects. Important structural differences are found. In its minimum energy configuration, the Be atom in the $V_{\mathrm{N}^{-}}-\mathrm{Be}_{\mathrm{Ga}}$ complex lies nearly in the same plane as the three equivalent $\mathrm{N}$ atoms nearest to it. Thus, it has shorter Be- $\mathrm{N}$ bonds than the Ga- $\mathrm{N}$ distance in the bulk crystal, while the $\mathrm{Mg}$ atom in the $V_{\mathrm{N}}-\mathrm{Mg}_{\mathrm{Ga}}$ complex occupies a position closer the lattice site of the $\mathrm{Ga}$ atom it replaces. Hence, the $V_{\mathrm{N}}-\mathrm{Be}_{\mathrm{Ga}}$ complex has a larger open volume than the $V_{\mathrm{N}}-\mathrm{Mg}_{\mathrm{Ga}}$ complex. This is consistent with positron annihilation experiments [Saarinen et al., J. Cryst. Growth 246, 281 (2002); Hautakangas et al., Phys. Rev. Lett. 90, 137402 (2003)]. The frequency of the highest local vibrational mode of the $V_{\mathrm{N}}-\mathrm{Be}_{\mathrm{Ga}}$ center is calculated to be within $3-4 \%$ of an infrared absorption line detected in Be-doped $\mathrm{GaN}$ [Clerjaud (private communication)].
\end{abstract}

DOI: 10.1103/PhysRevB.68.205209

PACS number(s): 61.72.Bb, 61.72.Ji, 61.72.Vv, 71.15.Nc

\section{INTRODUCTION}

Gallium nitride that has not been deliberately doped usually exhibits slightly $n$-type conducting behavior due to residual oxygen and silicon donors. The concentration of electrons can be readily increased by the addition of more oxygen or silicon. Unfortunately, it is more difficult to obtain $p$-type material. Magnesium acceptors have a relatively large activation energy, while beryllium-doped $\mathrm{GaN}$ is so heavily compensated that the material is only "semi-insulating." Five defects are considered in the work reported here as part of our continued investigation into the problems associated with doping GaN. These are the two vacancy-acceptor complexes $V_{\mathrm{N}}-\mathrm{Be}_{\mathrm{Ga}}$ and $V_{\mathrm{N}}-\mathrm{Mg}_{\mathrm{Ga}}$ and their constituents, the nitrogen vacancy $V_{\mathrm{N}}$, the substitutional beryllium acceptor $\mathrm{Be}_{\mathrm{Ga}}$, and the substitutional magnesium acceptor $\mathrm{Mg}_{\mathrm{Ga}}$. The results for $\mathrm{Be}_{\mathrm{Ga}}$ and $\mathrm{Mg}_{\mathrm{Ga}}$ are carried forward from our previous work. ${ }^{1}$ These two defects have simple structures in both their neutral and -1 charge states: the $\mathrm{Be}$ and $\mathrm{Mg}$ atoms in their minimum energy configuration are situated very close to the lattice site of the $\mathrm{Ga}$ atom that they replace. No other local minima were found.

A conclusion of the earlier work is that $\mathrm{Be}_{\mathrm{Ga}}$ defects have relatively long and somewhat weaker bonds to their four neighboring $\mathrm{N}$ atoms compared with those in a material such as $\mathrm{Be}_{3} \mathrm{~N}_{2}$. This results in very nearly equal frequencies for the highest local vibrational modes (LVMs) of $\mathrm{Be}_{\mathrm{Ga}}$ and $\mathrm{Mg}_{\mathrm{Ga}}$ defects in $\mathrm{GaN}$, rather than being more nearly proportional to $\sqrt{1 / m}$, where $m$ is the atomic mass. Thus, in the case of $\mathrm{Be}_{\mathrm{Ga}}$, the reduction in the frequencies of its LVMs due to having streched, weakened bonds outweighs the increase from having a smaller mass. The frequencies of the LVMs of both $\mathrm{Be}_{\mathrm{Ga}}$ and $\mathrm{Mg}_{\mathrm{Ga}}$ are below the $A_{1}\left(\mathrm{LO}, 735 \mathrm{~cm}^{-1}\right)$ phonon frequency of GaN, yet are in bands where the bulk phonon density of states are low, thus allowing the modes for $\mathrm{Mg}_{\mathrm{Ga}}$ defects in $\mathrm{GaN}$ to be observed by Raman experiments scattering. ${ }^{2-5}$. It is quite likely, therefore, that the modes for $\mathrm{Be}_{\mathrm{Ga}}$ defects could also be detected by the same technique. In the previous work, it was also suggested that $\mathrm{Be}_{\mathrm{Ga}}$ could combine with $V_{\mathrm{N}}$ to produce $V_{\mathrm{N}}-\mathrm{Be}_{\mathrm{Ga}}$ complexes that would have a larger open volume than $V_{\mathrm{N}}$ alone, and a higher frequency LVM than $\mathrm{Be}_{\mathrm{Ga}}$ by virtue of the $\mathrm{Be}$ atom in the defect having a more planar bonding arrangement with its three $\mathrm{N}$ atom neighbors. This could possibly explain the results of positron $\left(e^{+}\right)$annihilation experiments in which vacancies are found in Be-doped $\mathrm{GaN}$ that have a positron lifetime of $220 \pm 10 \mathrm{ps}$, which is more than that expected for bulk GaN (160-170 ps) and isolated $V_{\mathrm{N}}(160-180 \mathrm{ps})$ but slightly less than that measured for $V_{\mathrm{Ga}}(235 \pm 5 \mathrm{ps}){ }^{6}$

Moreover, there is a LVM that has been found in Bedoped GaN with a frequency $789 \mathrm{~cm}^{-1}$, and that is uncorrelated in amplitude with higher modes. ${ }^{1,7}$ This would be consistent with a Be-N bond length that is shorter than the one that isolated $\mathrm{Be}_{\mathrm{Ga}}$ defects have, as would be the case if the Be atom in a $V_{\mathrm{N}}-\mathrm{Be}_{\mathrm{Ga}}$ complex assumed a more planar, threefold-coordinated configuration. The positron annihilation experiments also find that in heavily $\mathrm{Mg}$-doped, $p$-type $\mathrm{GaN}$, there is a positron lifetime component $\approx 165 \pm 1 \mathrm{ps}$ 
(Ref. 6) It is not possible to say whether this is due to annihilation of $e^{+}$in the bulk crystal or is due to an unresolved combination of annihilation in the bulk and at $V_{\mathrm{N}^{-}} \mathrm{Mg}_{\mathrm{Ga}}$ complexes. In latter work, the positron lifetime for $V_{\mathrm{N}}-\mathrm{Mg}_{\mathrm{Ga}}$ complexes has been measured to be $180 \pm 5$ ps. $^{8}$

Neutral $V_{\mathrm{N}}$ defects have a singly occupied $t_{2}$ state that is thought to be resonant with the conduction band, and a doubly occupied $a_{1}$ state that is probably resonant with the valence band. ${ }^{9,10}$ Thus, $V_{\mathrm{N}}$ is expected to be an autoionizing effective-mass-like donor with +1 charge. Consequently, Coulomb repulsion will render it inoperative as a trap for $e^{+}$. It has been suggested that the $V_{\mathrm{N}}$ defect can exist in a +3 charge state that is stabilized by an outward relaxation of the neighboring Ga atoms. ${ }^{11}$ However, there is good reason to believe that the +3 state of $V_{\mathrm{N}}$ is only an artifact of the supercell method where the corrections required to treat charged supercells become more problematic for high charge states. Specifically, when ionization energies are calculated purely from total energies within the supercell formalism an additional term called the Madelung correction should strictly be applied. Its effect is to push donor levels deeper. Unfortunately, its convergence with supercell size is poor, and it involves parameters that are not possible to determine accurately, thus energies are often quoted without this correction. If a "suitable" reference state is available then ionization energies relative to it can be calculated more reliably than absolute values as the Madelung terms approximately cancel. As is often the case, for the defects that we are interested in, there is no comparable state of similar electronic character that can be used as a reference. For further discussion on this subject see, for example, Ref. 12.

By analogy with $V_{\mathrm{N}}$, we expect $V_{\mathrm{N}}-\mathrm{Be}_{\mathrm{Ga}}$ and $V_{\mathrm{N}}-\mathrm{Mg}_{\mathrm{Ga}}$ to be neutral defects, but may possibly exist in +2 charge states depending on exactly where the levels are in relation to the top of the valence band.

Thus, the aim of this work is to model in detail the properties of these defects by an accurate density-functional theory method within the formalism of the local-density approximation, to examine the validity of the ideas presented above.

\section{METHOD}

The total energies of supercells are calculated using a program package based on self-consistent local-densityfunctional theory, AIMPRO. ${ }^{13,14}$ It can provide detailed information about the structure of defects, their formation energies, electronic bandstructure, local vibrational modes, etc. The method uses a Gaussian basis set to describe the Kohn-Sham wave functions of the valence electrons, while the charge density is represented by a plane wave basis in reciprocal space, together with the Monkhorst-Pack (MP) scheme to sample the band structure. ${ }^{15}$ Core electrons are replaced by a pseudopotential based on the Troullier-Martins scheme. ${ }^{16}$ The semicore Ga $3 d$ electrons can be included explicitly in the valence orbitals. However, for the majority of calculations, we apply instead a nonlinear core correction to include their effects approximately. ${ }^{17}$ Selected tests are then performed to ensure that the approximation is valid for the particular problem under consideration. When calculating local vibrational modes, $a b$ initio second derivatives of energy are calculated for selected atoms in the immediate vicinity of a defect. We test whether the choice is adequate by extending the dynamical matrix to include "bulk" atoms by applying the Musgrave-Pople classical potential. ${ }^{18,19}$ The supercells used for these calculations contain 72 atoms in the ideal wurtzite-GaN crystal, where the lattice parameters $a$ $=3.1879 \AA$ and $c / a=1.6330$ give the minimum total energy for pure $\mathrm{GaN}$ by our method. Other details of the method are described in our previous work. ${ }^{1}$

\section{RESULTS AND DISCUSSION}

As discussed in the Introduction, the electronic structure of $V_{\mathrm{N}}, V_{\mathrm{N}}-\mathrm{Be}_{\mathrm{Ga}}$, and $V_{\mathrm{N}}-\mathrm{Mg}_{\mathrm{Ga}}$ is unusual compared with the majority of defects in that there are probably no levels in the bandgap. Purely for the record, and without applying the Madelung correction, the calculated electronic transition energies relative to one another are: $E\left(V_{\mathrm{N}}\right)^{+1+++}$ $\approx E\left(V_{\mathrm{N}^{-}}-\mathrm{Be}_{\mathrm{Ga}}\right)^{0 /++} \approx E\left(V_{\mathrm{N}}-\mathrm{Mg}_{\mathrm{Ga}}\right)^{0 /++} \approx\left[E\left(V_{\mathrm{N}}\right)^{0 /+}-2.2\right.$ $\mathrm{eV}]$ per electron. Recall that the $(0 /+)$ level of $V_{\mathrm{N}}$ appears to be an effective-mass state resonant with the bottom of the conduction band and note the Kohn-Sham bandgap $E_{K S}$ $\approx 2.2 \mathrm{eV}$. Also note that in the supercell formalism effectivemass states cannot be properly described as the extent of their wave function is very much greater than the size of the supercell. It must be stressed that we consider the validity of these electronic transition energies for the high charge states is extremely dubious and only two reliable conclusions can really be made about them. First, even if these states are real their high positive charge would prevent them from trapping $e^{+}$. Second, it is clear that under most circumstances, and possibly always, they are below the Fermi level $\mu_{e}$. Therefore, $V_{\mathrm{N}}$ will be in the +1 state, and $V_{\mathrm{N}}-\mathrm{Be}_{\mathrm{Ga}}$ and $V_{\mathrm{N}}-\mathrm{Mg}_{\mathrm{Ga}}$ will be neutral. This will certainly be the case in semiinsulating material where $\mu_{e}$ is near mid gap.

In terms of energy, both the $V_{\mathrm{N}}-\mathrm{Be}_{\mathrm{Ga}}$ and the $V_{\mathrm{N}}-\mathrm{Mg}_{\mathrm{Ga}}$ complexes are bound with respect to their isolated components. The energy for the reaction $\mathrm{Be}_{\mathrm{Ga}}^{-}+V_{\mathrm{N}}^{+} \rightarrow V_{\mathrm{N}}-\mathrm{Be}_{\mathrm{Ga}}$ $+\mathrm{GaN}$ is estimated to be $0.86 \mathrm{eV}$. When the acceptor is $\mathrm{Mg}$, the reaction $\mathrm{Mg}_{\mathrm{Ga}}^{-}+V_{\mathrm{N}}^{+} \rightarrow V_{\mathrm{N}}-\mathrm{Mg}_{\mathrm{Ga}}+\mathrm{GaN}$ yields about 0.53 $\mathrm{eV}$. The orientation of the defect makes no significant difference to the binding energy. These defects might form easily during growth: it only requires that a $\mathrm{N}$ atom fails to occupy one of the four sites next to a $\mathrm{Be}_{\mathrm{Ga}}$ or $\mathrm{Mg}_{\mathrm{Ga}}$ atom at or near the surface of a growing crystal. The fact this creates an energetically favorable complex makes the process all the more likely. A second Be acceptor binds to a $V_{\mathrm{N}^{-}}-\mathrm{Be}_{\mathrm{Ga}}$ complex so that the reaction $\mathrm{Be}_{\mathrm{Ga}}^{-}+V_{\mathrm{N}^{-}}-\mathrm{Be}_{\mathrm{Ga}} \rightarrow\left(V_{\mathrm{N}^{-}}\left(\mathrm{Be}_{\mathrm{Ga}}\right)_{2}\right)^{-}$ $+\mathrm{GaN}$ yields a further $0.77 \mathrm{eV}$. To create this complex it requires that two $\mathrm{Be}$ atoms occupy neighboring $\mathrm{Ga}$ sites. If the $\mathrm{Be}$ atoms are randomly distributed, then the concentration of $\left(V_{\mathrm{N}^{-}}\left(\mathrm{Be} \mathrm{Ga}_{\mathrm{Ga}}\right)^{-}\right.$will be low. A concentration closer to the equilibrium value for this defect might be achieved in the right growth conditions if a second $\mathrm{Be}$ atom is able to migrate to an existing $V_{\mathrm{N}}-\mathrm{Be}_{\mathrm{Ga}}$ complex and displace one of the neighboring $\mathrm{Ga}$ atoms. However, the activation energies of such processes need to be sufficiently low for them to occur 


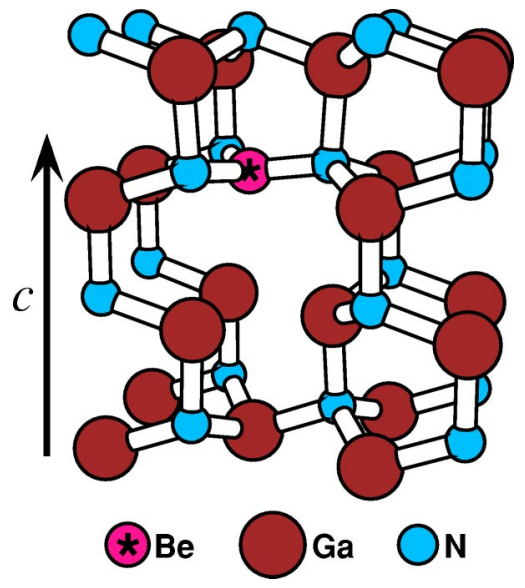
$\mathrm{GaN}$

FIG. 1. (Color online) Structure of the $V_{\mathrm{N}}-\mathrm{Be}_{\mathrm{Ga}}$ complex in

otherwise the system will remain far from equilibrium. The same remarks apply to the decomposition of these complexes into their isolated components. An order of magnitude estimate for the equilibrium concentration ratio $\left[V_{\mathrm{N}^{-}} \mathrm{Be}_{\mathrm{Ga}}\right] /\left[\left(V_{\mathrm{N}^{-}}\left(\mathrm{Be}_{\mathrm{Ga}}\right)_{2}\right)^{-}\right]$is in the range $\sim 1-1000$ for a growth temperature $T \approx 1500{ }^{\circ} \mathrm{C}$ and Be concentration [Be] $\sim 10^{21}-10^{19} \mathrm{~cm}^{-3}$. These figures are based on typical values for bulk GaN crystallized from liquid $\mathrm{Ga}$ and $\mathrm{N}_{2}$ gas at high pressures and temperatures. The specimens used in the infrared spectroscopy experiments and some of the positron annihilation experiments described previously were of this type. Lower $T$ or larger [Be] gives a smaller ratio. The corresponding ratio for $\mathrm{Mg}$ is perhaps $\sim 10$ times bigger.

The structure of a $V_{\mathrm{N}}-\mathrm{Be}_{\mathrm{Ga}}$ complex in its minimum energy configuration optimized by the AIMPRO method is illustrated in Fig. 1. Both this defect and the $V_{\mathrm{N}}-\mathrm{Mg}_{\mathrm{Ga}}$ complex have a similar overall appearance, but differ significantly in the amount that the atoms are displaced from the lattice sites of $\mathrm{Ga}$ and $\mathrm{N}$ atoms in bulk GaN. The Be atom in the $V_{\mathrm{N}}-\mathrm{Be}_{\mathrm{Ga}}$ complex lies nearly in the same plane as the three equivalent $\mathrm{N}$ atoms nearest to it, and about $0.42 \AA$ from the lattice site of the $\mathrm{Ga}$ atom that it replaces. The three $\mathrm{N}$ atoms closest to the Be atom are also shifted slightly from their normal positions towards the Be atom so that, combined with the shift of the $\mathrm{Be}$ atom along the $c$ direction, it makes the $\mathrm{Be}-\mathrm{N}$ distance about $13.2 \%$ less than the Ga-N distance in the bulk crystal. The $V_{\mathrm{N}}-\mathrm{Mg}_{\mathrm{Ga}}$ complex, however, has much smaller displacements of the atoms away from bulk lattice positions. The $\mathrm{Mg}$ atom is situated approximately $0.11 \AA$ further from the vacancy than the Ga lattice site, and the bonds to the three neighboring $\mathrm{N}$ atoms are only about $0.6 \%$ shorter than the normal, bulk $\mathrm{Ga}-\mathrm{N}$ distance. The atomic displacements are even smaller for the singly charged nitrogen vacancy $V_{\mathrm{N}}^{+}$, and are towards the vacancy rather than away from it. The unique $\mathrm{Ga}$ atom moves less than $0.01 \AA$, and the Ga-N distance to the three nearest $\mathrm{N}$ atoms is $0.2 \%$ longer than the bulk value. Table I contains details of these results together with others carried forward from our previous work. ${ }^{1}$

The dynamical matrix used to calculate the local vibrational modes of defects is constructed in two ways. In the first case, it only contains second derivatives of energy for a
TABLE I. Structural information for all combinations of $A_{\mathrm{N}}-B_{\mathrm{Ga}}$ with $A=V, \mathrm{~N}$, and $B=\mathrm{Be}, \mathrm{Mg}, \mathrm{Ga}$ in GaN calculated by the AIMPRO method. The distance $\delta_{c}$ is the displacement of atom $B$ in the $c$ direction. See also Fig. 1 , where $A=V$ and $B=$ Be.

\begin{tabular}{lccccccc}
\hline \hline$A$ & $B$ & Structure & $A-B(\AA)$ & $B-\mathrm{N}(\AA)$ & $\delta_{c}(\AA)$ & $\widehat{A B N}$ & $\widehat{\mathrm{NBN}}$ \\
\hline$V$ & $\mathrm{Be}$ & $V_{\mathrm{N}}-\mathrm{Be}_{\mathrm{Ga}}$ & & 1.695 & +0.420 & $96.3^{\circ}$ & $118.8^{\circ}$ \\
$V$ & $\mathrm{Mg}$ & $V_{\mathrm{N}}-\mathrm{Mg}_{\mathrm{Ga}}$ & & 1.940 & +0.115 & $105.5^{\circ}$ & $113.1^{\circ}$ \\
$V$ & $\mathrm{Ga}$ & $V_{\mathrm{N}}^{+}$ & & 1.939 & -0.006 & $109.0^{\circ}$ & $109.9^{\circ}$ \\
$\mathrm{N}$ & $\mathrm{Be}$ & $\mathrm{Be}$ & 1.802 & 1.805 & +0.042 & $109.3^{\circ}$ & $109.6^{\circ}$ \\
$\mathrm{N}$ & $\mathrm{Mg}$ & $\mathrm{Mg}_{\mathrm{Ga}}^{-}$ & 1.999 & 1.981 & +0.062 & $109.0^{\circ}$ & $109.9^{\circ}$ \\
$\mathrm{N}$ & $\mathrm{Ga}$ & $\mathrm{Bulk} \mathrm{GaN}$ & 1.955 & 1.952 & 0.000 & $109.5^{\circ}$ & $109.5^{\circ}$ \\
\hline \hline
\end{tabular}

few atoms calculated numerically using the analytic first derivatives. For the $V_{\mathrm{N}}-\mathrm{Be}_{\mathrm{Ga}}$ and $V_{\mathrm{N}}-\mathrm{Mg}_{\mathrm{Ga}}$ complexes in $\mathrm{GaN}$, these are the impurity atom, its three nearest nitrogen neighbors, and the three gallium atoms that are closest to the vacancy. The modes given by this method are then constrained to be completely localized on the defect atoms, and are not masked by bulk phonons, but it ignores dynamical interactions with other atoms. In the second case, the dynamical matrix is extended to include the contributions from other, more distant atoms by applying the Musgrave-Pople potential (MPP). The parameters for the potential are fitted to total energies for bulk GaN given by the AIMPRO method. If the choice of atoms for which $a b$ initio second derivatives are calculated is adequate, then these contributions should have very little effect on the frequencies of local modes, and thus act only as a check on and refinement to the method. However, local modes that are resonant with bulk phonons can no longer be distinguished.

The local vibrational modes for $V_{\mathrm{N}}-\mathrm{Be}_{\mathrm{Ga}}$ and $V_{\mathrm{N}}-\mathrm{Mg}_{\mathrm{Ga}}$ complexes in GaN calculated by the AIMPRO method are given in Table II. The results given are restricted to the higher frequencies of the centers, where the vibrations are mainly confined to the impurity $\mathrm{Be}$ and $\mathrm{Mg}$ atoms, and their three nitrogen neighbors. With the exception of the highest mode of the $V_{\mathrm{N}^{-}}-\mathrm{Be}_{\mathrm{Ga}}$ defect, all the modes are lower than the $A_{1}$ (LO, $735 \mathrm{~cm}^{-1}$ ) phonon frequency of GaN. However, several are in the bulk phonon bandgap between $\approx 330$ and $550 \mathrm{~cm}^{-1}$. Some others have frequencies within a "window" in the bulk bands at $\approx 620-660 \mathrm{~cm}^{-1}$ where the density of states is small making it possible to observe local modes in this region by Raman scattering experiments.

TABLE II. Local vibrational modes for $V_{\mathrm{N}}-\mathrm{Be}_{\mathrm{Ga}}$ and $V_{\mathrm{N}^{-}}-\mathrm{Mg}_{\mathrm{Ga}}$ complexes in GaN calculated by the AIMPRO method both with and without the Musgrave-Pople potential (MPP). When the MPP is applied local modes that are resonant with bulk phonon bands cannot be distinguished.

\begin{tabular}{lccccccccc}
\hline \hline Defect & MPP & \multicolumn{8}{c}{ Frequencies $\left(\mathrm{cm}^{-1}\right)$} \\
\hline$V_{\mathrm{N}}-\mathrm{Be}_{\mathrm{Ga}}$ & $\mathrm{No}$ & 422 & 431 & 568 & 583 & 608 & 640 & 671 & 756 \\
$V_{\mathrm{N}}-\mathrm{Be}_{\mathrm{Ga}}$ & Yes & 452 & 481 & 587 & 602 & 614 & & & 763 \\
$V_{\mathrm{N}}-\mathrm{Mg}_{\mathrm{Ga}}$ & $\mathrm{No}$ & & 307 & 601 & 613 & 648 & 656 & 671 & 683 \\
$V_{\mathrm{N}}-\mathrm{Mg}_{\mathrm{Ga}}$ & Yes & & 380 & 607 & 616 & & & & \\
\hline \hline
\end{tabular}


The two lowest modes given in Table II of the $V_{\mathrm{N}}-\mathrm{Be}_{\mathrm{Ga}}$ defect, as identified by their character and symmetry, are reversed in order of frequency when the MPP is applied. This is due to a much larger upward shift of the first mode relative to the second. In other words, the mode at $422 \mathrm{~cm}^{-1}$ increases to $481 \mathrm{~cm}^{-1}$, while the mode at $431 \mathrm{~cm}^{-1}$ changes by a much smaller amount to $452 \mathrm{~cm}^{-1}$. The lowest mode of $V_{\mathrm{N}}-\mathrm{Mg}_{\mathrm{Ga}}$ also suffers a large shift. Therefore, the frequencies of the modes at $422 / 481 \mathrm{~cm}^{-1}$ for $V_{\mathrm{N}^{-}} \mathrm{Be}_{\mathrm{Ga}}$, and $307 / 380 \mathrm{~cm}^{-1}$ for $V_{\mathrm{N}}-\mathrm{Mg}_{\mathrm{Ga}}$ cannot be considered reliable. Of the other modes that are not resonant with bulk phonon bands, the shifts in frequency when the MPP is applied are small and acceptable. In earlier work, the frequencies calculated for the LVMs of the $\mathrm{Mg}_{\mathrm{Ga}}$ defect were found to be in very good agreement with measured values. ${ }^{1}$ These include modes that are resonant with bulk phonon bands.

Several lines with frequencies above the bulk phonon bands, including one in particular at $789 \mathrm{~cm}^{-1}$, have been observed by infrared spectroscopy in Be-doped GaN. ${ }^{1,7} \mathrm{We}$ have identified the higher frequency LVMs as most probably being due to pairs of $\mathrm{Be}$ atoms sharing Ga-lattice sites, $(\mathrm{Be}-\mathrm{Be})_{\mathrm{Ga}}$. However, the line observed at $789 \mathrm{~cm}^{-1}$ is not correlated in amplitude with the other lines. A model based on a simple harmonic oscillator with empirical parameters suggests that this line might be due to isolated, substitutional beryllium, $\mathrm{Be}_{\mathrm{Ga}}$. Our calculations show that the highest mode of the $\mathrm{Be}_{\mathrm{Ga}}$ defect in fact has a relatively low frequency $\approx 663-679 \mathrm{~cm}^{-1}$ depending on the charge state. ${ }^{1}$ It also has $A_{1}$ symmetry. In infrared spectroscopy experiments, modes of this type are normally far too weak to be detected. The highest frequency mode of the $V_{\mathrm{N}}-\mathrm{Be}_{\mathrm{Ga}}$ complex predicted at $763 \mathrm{~cm}^{-1}$ has $E$ symmetry, and hence is expected to be infrared active.

\section{SUMMARY AND CONCLUSIONS}

While it is clear that the negatively charged gallium vacancy acts as an effective trap for $e^{+}$, the existence of vacancies detected by positron annihilation experiments in semi-insulating and $p$-type material appears to be less easy to explain. Under these conditions the formation energy of the gallium-vacancy is too large for it to exist in significant concentrations, while the nitrogen vacancy is excluded on the grounds of having a positive charge, and an open volume that is too small. The first clue for solving the problem is that the measured positron lifetime is significantly longer in Bedoped GaN than in Mg-doped GaN. Second, Be-doped material has a characteristic infrared absorption line at $789 \mathrm{~cm}^{-1}$ that is not correlated in intensity with other lines at higher frequencies. We propose that these experimental results can be explained by the presence of $V_{\mathrm{N}}-\mathrm{Be}_{\mathrm{Ga}}$ and $V_{\mathrm{N}}-\mathrm{Mg}_{\mathrm{Ga}}$ complexes in $\mathrm{GaN}$, and test our model using the AIMPRO local-density-functional theory method. According to this, we conclude that the difference in positron lifetimes between $\mathrm{Be}$-doped and $\mathrm{Mg}$-doped $\mathrm{GaN}$ is a consequence of the structural differences between the two types of complexes where the $V_{\mathrm{N}}-\mathrm{Be}_{\mathrm{Ga}}$ defect has a larger open volume than the $V_{\mathrm{N}}-\mathrm{Mg}_{\mathrm{Ga}}$ defect. The structures of the defects also have important consequences for their local vibrational modes. In particular, the presence of a nitrogen vacancy next to a substitutional $\mathrm{Be}$ atom raises the calculated frequency of the highest mode above the bulk phonon bands to a value that is within $3-4 \%$ of the infrared absorption line detected at $789 \mathrm{~cm}^{-1}$. The electronic structure of $V_{\mathrm{N}}-\mathrm{Be}_{\mathrm{Ga}}$ and $V_{\mathrm{N}}-\mathrm{Mg}_{\mathrm{Ga}}$ complexes is such that the defects will exist in a neutral charge state under most conditions. Therefore, their formation energy, and hence, their concentration is independent of the Fermi level $\mu_{e}$. The formation energy of these complexes is higher under N-rich conditions than Ga-rich conditions, as are the formation energies of interstitial-type defects, while the isolated acceptors $\mathrm{Be}_{\mathrm{Ga}}$ and $\mathrm{Mg}_{\mathrm{Ga}}$ behave in the opposite manner. Thus, N-rich stoichiometry tends to suppress the passivating defects, and favors acceptor formation.

\section{ACKNOWLEDGMENTS}

This research has been supported by the Engineering and Physical Sciences Research Council (EPSRC), U.K. (Contract No. GR/K05528) and the Academy of Finland through its Centers of Excellence Program 2000-2005. Computer resources have been provided by the Center for Scientific Computing in Finland (CSC) and Computer Services for Academic Research in the United Kingdom (CSAR). S.O. thanks VR in Sweden for providing financial support. The authors would also like to express their thanks to Kimmo Saarinen, Bernard Clerjaud, and Young-Joo Lee for helpful discussions.
*Email address: C.D.Latham@ex.ac.uk

${ }^{1}$ C.D. Latham, R.M. Nieminen, C.J. Fall, R. Jones, S. Öberg, and P.R. Briddon, Phys. Rev. B 67, 205206 (2003).

${ }^{2}$ A. Kaschner, H. Siegle, A. Hoffmann, C. Thomsen, U. Birkle, S. Einfeldt, and D. Hommel, MRS Internet J. Nitride Semicond. Res. 4S1, G3.57 (1999).

${ }^{3}$ A. Kaschner, H. Siegle, G. Kaczmarczyk, M. Straßburg, A. Hoffmann, C. Thomsen, U. Birkle, S. Einfeldt, and D. Hommel, Appl. Phys. Lett. 74, 3281 (1999).

${ }^{4}$ H. Harima, T. Inoue, S. Nakashima, M. Ishida, and M. Taneya, Appl. Phys. Lett. 75, 1383 (1999).

${ }^{5}$ H. Harima, T. Inoue, Y. Sone, S. Nakashima, M. Ishida, and M. Taneya, Phys. Status Solidi B 216, 789 (1999).
${ }^{6}$ K. Saarinen, V. Ranki, T. Suski, M. Bockowski, and I. Grzegory, J. Cryst. Growth 246, 281 (2002).

${ }^{7}$ B. Clerjaud (private communication). The Be-doped GaN used for these experiments was grown using a high-pressure, hightemperature method by UNIPRESS, Warsaw, Poland.

${ }^{8}$ S. Hautakangas, J. Oila, M. Alatalo, K. Saarinen, L. Liszkay, D. Seghier, and H.P. Gislason, Phys. Rev. Lett. 90, 137402 (2003).

${ }^{9}$ J. Neugebauer and C.G. Van de Walle, Phys. Rev. B 50, 8067 (1994).

${ }^{10}$ P. Boguslawski, E.L. Briggs, and J. Bernholc, Phys. Rev. B 51, 17 255 (1995).

${ }^{11}$ C.G. Van de Walle, C. Stampfl, and J. Neugebauer, J. Cryst. Growth 189-190, 505 (1998). 
${ }^{12}$ J. Coutinho, V.J.B. Torres, R. Jones, and P.R. Briddon, Phys. Rev. B 67, 035205 (2003).

${ }^{13}$ R. Jones and P.R. Briddon, The ab initio Cluster Method and the Dynamics of Defects in Semiconductors, Semiconductors and Semimetals, Vol. 51A (Academic Press, Boston, 1998), Chap. 6.

${ }^{14}$ J. Coutinho, R. Jones, P.R. Briddon, and S. Öberg, Phys. Rev. B 62, 10824 (2000).
${ }^{15}$ H.J. Monkhorst and J.D. Pack, Phys. Rev. B 13, 5188 (1976).

${ }^{16}$ N. Troullier and J.L. Martins, Phys. Rev. B 43, 1993 (1991).

${ }^{17}$ S.G. Louie, S. Froyen, and M.L. Cohen, Phys. Rev. B 26, 1738 (1982).

${ }^{18}$ M.J.P. Musgrave and J.A. Pople, Proc. R. Soc. London, Ser. A 268, 474 (1962).

${ }^{19}$ R. Jones, J. Phys. C 20, L271 (1987). 